\title{
Generalization of some extremal problems on non-overlapping domains with free poles
}

\begin{abstract}
Some results related to extremal problems with free poles on radial systems are generalized. They are obtained by applying the known methods of geometric function theory of complex variable. Sufficiently good numerical results for $\gamma$ are obtained.
\end{abstract}

1. Introduction. In geometric function theory of complex variable extremal problems on non-overlapping domains form the well-known classic direction. In the paper [1] Lavrent'ev posed and solved a problem of maximizing the product of conformal radii of two non-overlapping simply connected domains. Topics connected with the study of problems on nonoverlapping domains was developed in papers [1]-[21]. This paper summarizes some results obtained in [5], [2].

Let $\mathbb{N}, \mathbb{R}$ be the set of natural and real numbers, respectively, $\mathbb{C}$ be the complex plane, $\overline{\mathbb{C}}=\mathbb{C} \cup\{\infty\}$ be the one point compactification and $\mathbb{R}^{+}=(0, \infty)$.

Let $r(B, a)$ be an inner radius of a domain $B \subset \overline{\mathbb{C}}$ with respect to a point $a \in B$ (see [6], [13], [3]) and $\chi(t)=\frac{1}{2}\left(t+t^{-1}\right)$.

Let $n \in \mathbb{N}$. A set of points $A_{n}:=\left\{a_{k} \in \mathbb{C}: k=\overline{1, n}\right\}$ is called $\boldsymbol{n}$-radial system if $\left|a_{k}\right| \in \mathbb{R}^{+}, k=\overline{1, n}$, and $0=\arg a_{1}<\arg a_{2}<\ldots<\arg a_{n}<2 \pi$.

2010 Mathematics Subject Classification. 30C75.

Key words and phrases. Extremal problems on non-overlapping domains, inner radius, $n$-radial system of points, separating transformation. 
Denote

$$
\begin{gathered}
P_{k}\left(A_{n}\right):=\left\{w: \arg a_{k}<\arg w<\arg a_{k+1}\right\}, \\
\theta_{k}:=\arg a_{k}, \quad a_{n+1}:=a_{1}, \quad \theta_{n+1}:=2 \pi, \\
\alpha_{k}:=\frac{1}{\pi} \arg \frac{a_{k+1}}{a_{k}}, \quad \alpha_{n+1}:=\alpha_{1}, \quad k=\overline{1, n} .
\end{gathered}
$$

This work is based on application of separating transformation developed in [4]-[6]. For specific use of this method we consider a special system of conformal mappings. By $\zeta=\pi_{k}(w)=-i\left(e^{-i \theta_{k}} w\right)^{\frac{1}{\alpha_{k}}}, \quad k=\overline{1, n}$ we denote unique branch of multi-valued analytic function $\pi_{k}(w)$ performing univalent and conformal mapping $P_{k}\left(A_{n}\right)$ onto the right half plane $\operatorname{Re} \zeta>0$.

For an arbitrary $n$-radial system of points $A_{n}=\left\{a_{k}\right\}$ and $\gamma \in \mathbb{R}^{+}$we assume that

$$
\begin{aligned}
\mathcal{L}^{(\gamma)}\left(A_{n}\right):= & \prod_{k=1}^{n}\left[\chi\left(\left|\frac{a_{k}}{a_{k+1}}\right|^{\frac{1}{2 \alpha_{k}}}\right)\right]^{1-\frac{1}{2} \gamma \alpha_{k}^{2}} \cdot \prod_{k=1}^{n}\left|a_{k}\right|^{1+\frac{1}{4} \gamma\left(\alpha_{k}+\alpha_{k-1}\right)}, \\
& \mathcal{L}^{(0)}\left(A_{n}\right):=\prod_{k=1}^{n}\left[\chi\left(\left|\frac{a_{k}}{a_{k+1}}\right|^{\frac{1}{2 \alpha_{k}}}\right)\right] \cdot\left|a_{k}\right| .
\end{aligned}
$$

The class of $n$-radial systems of points for which $\mathcal{L}^{(\gamma)}\left(A_{n}\right)=1\left(\mathcal{L}^{(0)}\left(A_{n}\right)=1\right)$ automatically includes all systems with $n$ different points located on the unit circle.

The main purpose of this work is to obtain exact upper estimates for the functionals

$$
\begin{gathered}
J_{n}(\gamma)=r^{\gamma}\left(B_{0}, 0\right) \prod_{k=1}^{n} r\left(B_{k}, a_{k}\right), \\
I_{n}(\gamma)=\left[r\left(B_{0}, 0\right) r\left(B_{\infty}, \infty\right)\right]^{\gamma} \prod_{k=1}^{n} r\left(B_{k}, a_{k}\right),
\end{gathered}
$$

where $\gamma \in \mathbb{R}^{+}, A_{n}=\left\{a_{k}\right\}_{k=1}^{n}$ is $n$-radial system of points, $a_{0}=0$, and $\left\{B_{k}\right\}_{k=0}^{n}$ is a system of non-overlapping domains (i.e. $B_{p} \cap B_{j}=\emptyset$ if $p \neq j$ ) such that $a_{k} \in B_{k}, k=\overline{0, n}$.

\section{Main results.}

Theorem 1. Let $n \in \mathbb{N}, n \geq 2$ and $\gamma \in(0,1]$. Then for any $n$-radial system of points $A_{n}=\left\{a_{k}\right\}_{k=1}^{n}$ such that $\mathcal{L}^{(\gamma)}\left(A_{n}\right)=1$ and any system of non-overlapping domains $B_{k}, a_{k} \in B_{k} \subset \overline{\mathbb{C}}, k=\overline{1, n}, a_{0}=0 \in B_{0}$ we have the inequality

$$
J_{n}(\gamma) \leq \frac{4^{n+\frac{\gamma}{n}} \gamma^{\frac{\gamma}{n}} n^{n}}{\left(n^{2}-\gamma\right)^{n+\frac{\gamma}{n}}}\left(\frac{n-\sqrt{\gamma}}{n+\sqrt{\gamma}}\right)^{2 \sqrt{\gamma}}
$$


Equality in (2) is achieved when $a_{k}$ and $B_{k}, k=\overline{0, n}$ are, respectively, poles and circular domains of the quadratic differential

$$
Q(w) d w^{2}=-\frac{\left(n^{2}-\gamma\right) w^{n}+\gamma}{w^{2}\left(w^{n}-1\right)^{2}} d w^{2} .
$$

Theorem 2. Let $n \in \mathbb{N}, n \geq 2$ and $\gamma=\frac{1}{2}$. Then for any $n$-radial system of points $A_{n}=\left\{a_{k}\right\}_{k=1}^{n}$ such that $\mathcal{L}^{(0)}\left(A_{n}\right)=1$ and any system of nonoverlapping domains $B_{k}, B_{0}, B_{\infty}, a_{k} \in B_{k} \subset \overline{\mathbb{C}}, k=\overline{1, n}, a_{0}=0 \in B_{0} \subset \overline{\mathbb{C}}$, $a_{\infty}=\infty \in B_{\infty} \subset \overline{\mathbb{C}}$ we have the inequality

$$
\left[r\left(B_{0}, 0\right) r\left(B_{\infty}, \infty\right)\right]^{\frac{1}{2}} \prod_{k=1}^{n} r\left(B_{k}, a_{k}\right) \leq \frac{2^{2 n+\frac{1}{n}}}{\left(n^{2}-2\right)^{\frac{1}{n}+\frac{n}{2}}}\left(\frac{n-\sqrt{2}}{n+\sqrt{2}}\right)^{\sqrt{2}} .
$$

Equality in (3) is achieved, when $a_{k}$ and $B_{k}$ are, respectively, poles and circular domains of the quadratic differential

$$
Q(w) d w^{2}=-\frac{w^{2 n}+w^{n}\left(2 n^{2}-2\right)+1}{w^{2}\left(w^{n}-1\right)^{2}} d w^{2} .
$$

Theorem 3. Let $\gamma \in\left(0 ; \gamma_{2}^{0}\right], \gamma_{2}^{0}=1.1, \varepsilon=0.25$. Then for any 2-radial system of points $A_{2}=\left\{a_{k}\right\}_{k=1}^{2}$ such that $\mathcal{L}^{(\gamma)}\left(A_{2}\right)=1,1-\varepsilon<\left|a_{k}\right|<1+\varepsilon$, $k=\overline{1,2}$ and any system of non-overlapping domains $\left\{B_{k}\right\}_{k=0}^{2}, a_{k} \in B_{k}$, $k=\overline{0,2}, a_{0}=0 \in B_{0}$, we have the inequality

$$
r^{\gamma}\left(B_{0}, 0\right) \prod_{k=1}^{2} r\left(B_{k}, a_{k}\right) \leq r^{\gamma}\left(D_{0}, 0\right) \prod_{k=1}^{2} r\left(D_{k}, d_{k}\right),
$$

where $D_{k}, d_{k}, k=\overline{0,2}, d_{0}=0$, are circular domains and poles of the quadratic differential

$$
Q(w) d w^{2}=-\frac{(4-\gamma) w^{2}+\gamma}{w^{2}\left(w^{2}-1\right)^{2}} d w^{2}
$$

Proof of Theorem 1. We use the method due to Bakhtin [2]-[3] and properties of separating transformation (see [4]-[7], [3], [8]). We make separating transformation of domains $\left\{B_{k}\right\}_{k=1}^{n}$. Suppose

$$
P_{k}:=P_{k}\left(A_{n}\right):=\left\{w \in \mathbb{C} \backslash\{0\}: \theta_{k}<\arg w<\theta_{k+1}\right\} .
$$

Consider the introduced system of functions $\zeta=\pi_{k}(w)=-i\left(e^{-i \theta_{k}} w\right)^{\frac{1}{\alpha_{k}}}$, $k=\overline{1, n}$.

Let $\Omega_{k}^{(1)}, k=\overline{1, n}$ be a domain of the plane $\mathbb{C}_{\zeta}$ obtained by combining the connected component $\pi_{k}\left(B_{k} \cap \bar{P}_{k}\right)$ containing a point $\pi_{k}\left(a_{k}\right)$, with its symmetrical reflection with respect to the imaginary axis.

By $\Omega_{k}^{(2)}, k=\overline{1, n}$, we denote the domain of the plane $\mathbb{C}_{\zeta}$, obtained by combining the connected component $\pi_{k}\left(B_{k+1} \bigcap \bar{P}_{k}\right)$, containing the point 
$\pi_{k}\left(a_{k+1}\right)$, with its symmetrical reflection with respect to the imaginary axis, $B_{n+1}:=B_{1}, \pi_{n}\left(a_{n+1}\right):=\pi_{n}\left(a_{1}\right)$.

Besides, by $\Omega_{k}^{(0)}$ we denote the domain of $\mathbb{C}_{\zeta}$, obtained by combining the connected component $\pi_{k}\left(B_{0} \cap \bar{P}_{k}\right)$, containing the point $\zeta=0$, with its symmetrical reflection with respect to the imaginary axis. Denote $\pi_{k}\left(a_{k}\right)$ : $=\omega_{k}^{(1)}, \pi_{k}\left(a_{k+1}\right):=\omega_{k}^{(2)}, k=\overline{1, n}, \pi_{n}\left(a_{n+1}\right):=\omega_{n}^{(2)}$.

The definition of $\pi_{k}$ implies that

$$
\begin{gathered}
\left|\pi_{k}(w)-\omega_{k}^{(1)}\right| \sim \frac{1}{\alpha_{k}}\left|a_{k}\right|^{\frac{1}{\alpha_{k}}-1} \cdot\left|w-a_{k}\right|, \quad w \rightarrow a_{k}, \quad w \in \overline{P_{k}}, \\
\left|\pi_{k}(w)-\omega_{k}^{(2)}\right| \sim \frac{1}{\alpha_{k}}\left|a_{k+1}\right|^{\frac{1}{\alpha_{k}}-1} \cdot\left|w-a_{k+1}\right|, \quad w \rightarrow a_{k+1}, \quad w \in \overline{P_{k}}, \\
\left|\pi_{k}(w)\right| \sim|w|^{\frac{1}{\alpha_{k}}}, \quad w \rightarrow 0, \quad w \in \overline{P_{k}} .
\end{gathered}
$$

Then, using results of papers [4]-[7], [3], we obtain the inequalities

$$
\begin{gathered}
r\left(B_{k}, a_{k}\right) \leq\left[\frac{r\left(\Omega_{k}^{(1)}, \omega_{k}^{(1)}\right) \cdot r\left(\Omega_{k}^{(2)}, \omega_{k}^{(2)}\right)}{\frac{1}{\alpha_{k}}\left|a_{k}\right|^{\frac{1}{\alpha_{k}}-1} \cdot \frac{1}{\alpha_{k-1}}\left|a_{k}\right|^{\frac{1}{\alpha_{k-1}}-1}}\right]^{\frac{1}{2}}, \\
k=\overline{1, n}, \quad \Omega_{0}^{(2)}:=\Omega_{n}^{(2)}, \quad \omega_{0}^{(2)}:=\omega_{n}^{(2)} \\
r\left(B_{0}, 0\right) \leq\left[\prod_{k=1}^{n} r^{\alpha_{k}^{2}}\left(\Omega_{k}^{(0)}, 0\right)\right]^{\frac{1}{2}} .
\end{gathered}
$$

Repeating arguments given in [3] in the proof of Theorem 5.2.1 and taking into account introduced sets of domains $\left\{P_{k}\right\}_{k=1}^{n}$, functions $\left\{\pi_{k}\right\}_{k=1}^{n}$ and numbers $\left\{\theta_{k}\right\}_{k=1}^{n}$, we obtain the following inequality for the investigated functional (1):

$$
\begin{aligned}
J_{n}(\gamma) \leq & \prod_{k=1}^{n}\left[r\left(\Omega_{k}^{(0)}, 0\right)\right]^{\frac{\alpha_{k}^{2}}{2} \gamma} \cdot \prod_{k=1}^{n}\left[\frac{r\left(\Omega_{k-1}^{(2)}, \omega_{k-1}^{(2)}\right) r\left(\Omega_{k}^{(1)}, \omega_{k}^{(1)}\right)}{\frac{1}{\alpha_{k-1} \cdot \alpha_{k}}\left|a_{k}\right|^{\frac{1}{\alpha_{k-1}}-1} \cdot\left|a_{k}\right|^{\frac{1}{\alpha_{k}}-1}}\right]^{\frac{1}{2}} \\
= & \prod_{k=1}^{n} \alpha_{k} \cdot \prod_{k=1}^{n} \frac{\left|a_{k}\right|}{\left|a_{k} a_{k+1}\right|^{\frac{1}{2 \alpha_{k}}}} \\
& \times\left[\prod_{k=1}^{n} r^{\gamma \alpha_{k}^{2}}\left(\Omega_{k}^{(0)}, 0\right) \prod_{k=1}^{n} r\left(\Omega_{k}^{(1)}, \omega_{k}^{(1)}\right) r\left(\Omega_{k}^{(2)}, \omega_{k}^{(2)}\right)\right]^{\frac{1}{2}} .
\end{aligned}
$$

Expression in parentheses of the last formula in (6) is a product of the functional $r^{\beta^{2}}\left(\Omega_{k}^{(0)}, 0\right) r\left(\Omega_{k}^{(1)}, \omega_{k}^{(1)}\right) r\left(\Omega_{k}^{(2)}, \omega_{k}^{(2)}\right)$ on triples of domains $\left(\Omega_{k}^{(0)}\right.$, $\left.\Omega_{k}^{(1)}, \Omega_{k}^{(2)}\right)$ of the plane $\mathbb{C}_{\zeta}$. 
It is known [15] that the functional

$$
Y_{3}\left(\sigma_{1}, \sigma_{2}, \sigma_{3}\right)=\frac{r^{\sigma_{1}}\left(D_{1}, d_{1}\right) \cdot r^{\sigma_{2}}\left(D_{2}, d_{2}\right) \cdot r^{\sigma_{3}}\left(D_{3}, d_{3}\right)}{\left|d_{1}-d_{2}\right|^{\sigma_{1}+\sigma_{2}-\sigma_{3}} \cdot\left|d_{1}-d_{3}\right|^{\sigma_{1}-\sigma_{2}+\sigma_{3}} \cdot\left|d_{2}-d_{3}\right|^{-\sigma_{1}+\sigma_{2}+\sigma_{3}}},
$$

$\sigma_{k} \in \mathbb{R}^{+}, d_{k} \in D_{k} \subset \overline{\mathbb{C}}, D_{k} \cap D_{p}=\varnothing, k=1,2,3, p=1,2,3, k \neq p$, is invariant under all conformal automorphisms of the complex plane $\overline{\mathbb{C}}$.

With this relation in mind, the following estimate holds:

$$
\begin{aligned}
& J_{n}(\gamma) \leq\left(\prod_{k=1}^{n} \alpha_{k}\right) \cdot \prod_{k=1}^{n} \frac{\left|a_{k}\right|}{\left|a_{k} a_{k+1}\right|^{\frac{1}{2 \alpha_{k}}}} \\
& \times\left\{\prod_{k=1}^{n} \frac{r^{\gamma \alpha_{k}^{2}}\left(\Omega_{k}^{(0)}, 0\right) \cdot r\left(\Omega_{k}^{(1)}, \omega_{k}^{(1)}\right) \cdot r\left(\Omega_{k}^{(2)}, \omega_{k}^{(2)}\right)}{\left|\omega_{k}^{(1)} \cdot \omega_{k}^{(2)}\right| \gamma \alpha_{k}^{2}\left|\omega_{k}^{(1)}-\omega_{k}^{(2)}\right|^{2-\gamma \alpha_{k}^{2}}}\right\}^{\frac{1}{2}} \\
& \times\left[\prod_{k=1}^{n}\left|\omega_{k}^{(1)} \cdot \omega_{k}^{(2)}\right|^{\gamma \alpha_{k}^{2}}\left|\omega_{k}^{(1)}-\omega_{k}^{(2)}\right|^{2-\gamma \alpha_{k}^{2}}\right]^{\frac{1}{2}} \text {. }
\end{aligned}
$$

Note that $\left|\omega_{k}^{(1)}\right|=\left|a_{k}\right|^{\frac{1}{\alpha_{k}}},\left|\omega_{k}^{(2)}\right|=\left|a_{k+1}\right|^{\frac{1}{\alpha_{k}}},\left|\omega_{k}^{(1)}-\omega_{k}^{(2)}\right|=\left|a_{k}\right|^{\frac{1}{\alpha_{k}}}+$ $\left|a_{k+1}\right|^{\frac{1}{\alpha_{k}}}$.

Taking into account these equalities, we obtain

$$
\begin{aligned}
& J_{n}(\gamma) \leq\left(\prod_{k=1}^{n} \alpha_{k}\right) \cdot \prod_{k=1}^{n} \frac{\left|a_{k}\right|}{\left|a_{k} a_{k+1}\right|^{\frac{1}{2 \alpha_{k}}}} \\
& \times\left(\prod_{k=1}^{n}\left|\omega_{k}^{(1)}-\omega_{k}^{(2)}\right|\right)\left(\prod_{k=1}^{n} \frac{\left|\omega_{k}^{(1)} \cdot \omega_{k}^{(2)}\right|}{\left|\omega_{k}^{(1)}-\omega_{k}^{(2)}\right|}\right)^{\frac{\gamma \alpha_{k}^{2}}{2}} \\
& \times\left\{\prod_{k=1}^{n} \frac{r^{\gamma \alpha_{k}^{2}}\left(\Omega_{k}^{(0)}, 0\right) \cdot r\left(\Omega_{k}^{(1)}, \omega_{k}^{(1)}\right) \cdot r\left(\Omega_{k}^{(2)}, \omega_{k}^{(2)}\right)}{\left|\omega_{k}^{(1)} \cdot \omega_{k}^{(2)}\right| \gamma \alpha_{k}^{2}\left|\omega_{k}^{(1)}-\omega_{k}^{(2)}\right|^{2-\gamma \alpha_{k}^{2}}}\right\}^{\frac{1}{2}} \\
& =2^{n} \cdot\left(\prod_{k=1}^{n} \alpha_{k}\right) \cdot \prod_{k=1}^{n} \chi\left(\left|\frac{a_{k}}{a_{k+1}}\right|^{\frac{1}{2 \alpha_{k}}}\right)\left|a_{k}\right| \\
& \times 2^{-\frac{\gamma}{2} \sum_{k=1}^{n} \alpha_{k}}\left[\prod_{k=1}^{n} \chi\left(\left|\frac{a_{k}}{a_{k+1}}\right|^{\frac{1}{2 \alpha_{k}}}\right)\right]^{-\frac{\gamma \alpha_{k}^{2}}{2}}\left(\prod_{k=1}^{n}\left|\frac{a_{k+1}}{a_{k}}\right|\right)^{\frac{\gamma \alpha_{k}^{2}}{2}} \\
& \times\left\{\prod_{k=1}^{n} \frac{r^{\gamma \alpha_{k}^{2}}\left(\Omega_{k}^{(0)}, 0\right) \cdot r\left(\Omega_{k}^{(1)}, \omega_{k}^{(1)}\right) \cdot r\left(\Omega_{k}^{(2)}, \omega_{k}^{(2)}\right)}{\left|\omega_{k}^{(1)} \cdot \omega_{k}^{(2)}\right| \gamma \alpha_{k}^{2}\left|\omega_{k}^{(1)}-\omega_{k}^{(2)}\right|^{2-\gamma \alpha_{k}^{2}}}\right\}^{\frac{1}{2}}
\end{aligned}
$$




$$
\begin{aligned}
& =2^{n-\frac{\gamma}{2} \sum_{k=1}^{n} \alpha_{k}^{2}} \cdot\left(\prod_{k=1}^{n} \alpha_{k}\right) \cdot \prod_{k=1}^{n}\left[\chi\left(\left|\frac{a_{k}}{a_{k+1}}\right|^{\frac{1}{2 \alpha_{k}}}\right)\right]^{1-\frac{\gamma \alpha_{k}^{2}}{2}} \\
& \times \prod_{k=1}^{n}\left|a_{k}\right|^{1+\frac{1}{4} \gamma\left(\alpha_{k}+\alpha_{k-1}\right)} \\
& \times\left\{\prod_{k=1}^{n} \frac{r^{\gamma \alpha_{k}^{2}}\left(\Omega_{k}^{(0)}, 0\right) \cdot r\left(\Omega_{k}^{(1)}, \omega_{k}^{(1)}\right) \cdot r\left(\Omega_{k}^{(2)}, \omega_{k}^{(2)}\right)}{\left|\omega_{k}^{(1)} \cdot \omega_{k}^{(2)}\right|^{\gamma \alpha_{k}^{2}}\left|\omega_{k}^{(1)}-\omega_{k}^{(2)}\right|^{2-\gamma \alpha_{k}^{2}}}\right\}^{\frac{1}{2}} \\
& =2^{n-\frac{\gamma}{2} \sum_{k=1}^{n} \alpha_{k}^{2}} \cdot\left(\prod_{k=1}^{n} \alpha_{k}\right) \cdot \mathcal{L}^{(\gamma)}\left(A_{n}\right) \\
& \times\left\{\prod_{k=1}^{n} \frac{r^{\gamma \alpha_{k}^{2}}\left(\Omega_{k}^{(0)}, 0\right) \cdot r\left(\Omega_{k}^{(1)}, \omega_{k}^{(1)}\right) \cdot r\left(\Omega_{k}^{(2)}, \omega_{k}^{(2)}\right)}{\left|\omega_{k}^{(1)} \cdot \omega_{k}^{(2)}\right|^{\gamma \alpha_{k}^{2}}\left|\omega_{k}^{(1)}-\omega_{k}^{(2)}\right|^{2-\gamma \alpha_{k}^{2}}}\right\}^{\frac{1}{2}} .
\end{aligned}
$$

For each $k=\overline{1, n}$ we can easily define conformal automorphism $\zeta=T_{k}(z)$ of complex numbers of the plane $\overline{\mathbb{C}}$ such that $T_{k}(0)=0, T_{k}\left(\omega_{k}^{(s)}\right)=(-1)^{s} \cdot i$, $G_{k}^{(q)}:=T_{k}\left(\Omega_{k}^{(q)}\right), k=\overline{1, n}, s=1,2, q=0,1,2$.

Then

$$
\begin{aligned}
& \left\{\prod_{k=1}^{n} \frac{r^{\gamma \alpha_{k}^{2}}\left(\Omega_{k}^{(0)}, 0\right) \cdot r\left(\Omega_{k}^{(1)}, \omega_{k}^{(1)}\right) \cdot r\left(\Omega_{k}^{(2)}, \omega_{k}^{(2)}\right)}{\left.\left|\omega_{k}^{(1)} \cdot \omega_{k}^{(2)}\right| \gamma \alpha_{k}^{2}\left|\omega_{k}^{(1)}-\omega_{k}^{(2)}\right|^{2-\gamma \alpha_{k}^{2}}\right\}^{\frac{1}{2}}}\right. \\
& =\left\{\prod_{k=1}^{n} \frac{r^{\alpha_{k}^{2} \gamma}\left(G_{k}^{(0)}, 0\right) \cdot r\left(G_{k}^{(1)},-i\right) \cdot r\left(G_{k}^{(2)}, i\right)}{2^{2-\gamma \alpha_{k}^{2}}}\right\}^{\frac{1}{2}} .
\end{aligned}
$$

Then using results of [3], [15], we obtain

$$
\begin{aligned}
J_{n}(\gamma) & \leq 2^{n-\frac{\gamma}{2}} \sum_{k=1}^{n} \alpha_{k}^{2} \cdot\left(\prod_{k=1}^{n} \alpha_{k}\right) \cdot \mathcal{L}^{(\gamma)}\left(A_{n}\right) \\
& \times \prod_{k=1}^{n}\left\{\frac{r^{\alpha_{k}^{2} \gamma}\left(G_{k}^{(0)}, 0\right) \cdot r\left(G_{k}^{(1)},-i\right) \cdot r\left(G_{k}^{(2)}, i\right)}{2^{2-\gamma \alpha_{k}^{2}}}\right\}^{\frac{1}{2}} \\
& =2^{n-\frac{\gamma}{2}} \sum_{k=1}^{n} \alpha_{k}^{2}\left(\prod_{k=1}^{n} \alpha_{k}\right) \cdot \mathcal{L}^{(\gamma)}\left(A_{n}\right) \cdot 2^{-n+\frac{\gamma}{2} \sum_{k=1}^{n} \alpha_{k}^{2}}
\end{aligned}
$$




$$
\begin{gathered}
\times\left[\prod_{k=1}^{n} r^{\alpha_{k}^{2} \gamma}\left(G_{k}^{(0)}, 0\right) \cdot r\left(G_{k}^{(1)},-i\right) \cdot r\left(G_{k}^{(2)}, i\right)\right]^{\frac{1}{2}} \\
\leq\left(\prod_{k=1}^{n} \alpha_{k}\right) \cdot \mathcal{L}^{(\gamma)}\left(A_{n}\right) \cdot\left[\prod_{k=1}^{n} r^{\alpha_{k}^{2} \gamma}\left(G_{k}^{(0)}, 0\right) \cdot r\left(G_{k}^{(1)},-i\right) \cdot r\left(G_{k}^{(2)}, i\right)\right]^{\frac{1}{2}} .
\end{gathered}
$$

Following the paper [15], we have

$$
J_{n}(\gamma) \leq \gamma^{-\frac{n}{2}}\left[\prod_{k=1}^{n} \Psi\left(\beta_{k}\right)\right]^{1 / 2}
$$

where $\Psi(\beta)=2^{\beta^{2}+6} \cdot \beta^{\beta^{2}+2}(2-\beta)^{-\frac{1}{2}(2-\beta)^{2}} \cdot(2+\beta)^{-\frac{1}{2}(2+\beta)^{2}}, \beta \in[0,2]$.

Similarly to [5], we consider the next extremal problem:

$$
\prod_{k=1}^{n} \Psi\left(\beta_{k}\right) \rightarrow \sup ; \quad \sum_{k=1}^{n} \beta_{k}=2 \sqrt{\gamma}, \beta_{k}=\alpha_{k} \sqrt{\gamma}, 0<\beta_{k} \leq 2 .
$$

Necessary conditions have the form

$$
\frac{\Psi^{\prime}\left(\beta_{k}\right)}{\Psi\left(\beta_{k}\right)}=\frac{-\lambda}{\prod_{k=1}^{n} \Psi\left(\beta_{k}\right)}, \quad k=\overline{1, n} .
$$

We will show that all $\beta_{k}$ are equal. We investigate behavior of the function $F(\beta)=\frac{\Psi^{\prime}(\beta)}{\Psi(\beta)}=2 \beta \ln (2 \beta)+\frac{2}{\beta}+(2-\beta) \ln (2-\beta)-(2+\beta) \ln (2+\beta)$ on interval $\beta \in[0,2]$. It is strictly decreasing on the interval $\left(0 ; \beta_{0}\right], \beta_{0} \in(1.32 ; 1.33)$ and increasing on $\left[\beta_{0} ; 2\right)$. Then we use the method of the proof of Theorem 4 [5] and obtain that unique solution of the extremal problem is the point $\left(\frac{2}{n}, \ldots, \frac{2}{n}\right)$. Estimates $(4),(5),(7)$ yield inequality (2) of Theorem 1 . The case of equality is verified directly and Theorem 1 is proved.

Dubinin proved Theorem 1 if $\gamma=1$ and for any distinct points $a_{k}$ that lie on the unit circle and any non-overlapping domains $B_{k}$ (see [5], [8]).

Proof of Theorem 2. We retain all notation for separating transformation of domains introduced in the proof of Theorem 1 for domains $B_{k}$, $k=\overline{0, n}$. By $\Omega_{k}^{(\infty)}$ we denote the domain of plane $\mathbb{C}_{\zeta}$, obtained by combining the connected component $\pi_{k}\left(B_{\infty} \cap \bar{E}_{k}\right)$ containing the point $\zeta=\infty$ with its symmetrical reflection with respect to the imaginary axis. The family $\left\{\Omega_{k}^{(\infty)}\right\}_{k=1}^{n}$ is a result of separating transformation of an arbitrary domain $B_{\infty}, \infty \in B_{\infty} \subset \overline{\mathbb{C}}$ with respect to the families $\left\{P_{k}\right\}_{k=1}^{n}$ and $\left\{\pi_{k}\right\}_{k=1}^{n}$ at the point $\zeta=\infty$. 
By Theorem 2 in [5] we have

$$
r\left(B_{\infty}, \infty\right) \leq\left[\prod_{k=1}^{n} r^{\alpha_{k}^{2}}\left(\Omega_{k}^{(\infty)}, \infty\right)\right]^{\frac{1}{2}} .
$$

Using (4), (5), (8), we obtain

$$
\begin{aligned}
& {\left[r\left(B_{0}, 0\right) r\left(B_{\infty}, \infty\right)\right]^{\frac{1}{2}} \prod_{k=1}^{n} r\left(B_{k}, a_{k}\right) \leq 2^{n} \cdot\left(\prod_{k=1}^{n} \alpha_{k}\right) \cdot \mathcal{L}^{(0)}\left(A_{n}\right)} \\
& \quad \times\left[\prod_{k=1}^{n} r^{\alpha_{k}^{2} \gamma}\left(\Omega_{k}^{(0)}, 0\right) \cdot r^{\alpha_{k}^{2} \gamma}\left(\Omega_{k}^{(\infty)}, \infty\right) \cdot \frac{r\left(\Omega_{k}^{(1)}, \omega_{k}^{(1)}\right) \cdot r\left(\Omega_{k}^{(2)}, \omega_{k}^{(2)}\right)}{\left|\omega_{k}^{(1)}-\omega_{k}^{(2)}\right|^{2}}\right]^{\frac{1}{2}} .
\end{aligned}
$$

Theorem 6 in [5] gives

$$
\begin{aligned}
& {\left[r\left(B_{0}, 0\right) r\left(B_{\infty}, \infty\right)\right]^{\alpha_{k}^{2} \gamma} \cdot \frac{r\left(B_{1}, a_{1}\right) r\left(B_{2}, a_{2}\right)}{\left|a_{1}-a_{2}\right|^{2}} \leq \Psi(\beta)} \\
& \quad=\beta^{2 \beta^{2}} \cdot|1-\beta|^{-(1-\beta)^{2}} \cdot(1+\beta)^{-(1+\beta)^{2}}, \quad 0<\beta \leq \sqrt{2} .
\end{aligned}
$$

Inequality (9) was obtained by Dubinin using the results of Kolbina [15]. Similarly to [5], we consider the extremal problem:

$$
\prod_{k=1}^{n} \Psi\left(\beta_{k}\right) \rightarrow \sup ; \quad \sum_{k=1}^{n} \beta_{k}=\sqrt{2} .
$$

We introduce a function $F(\beta)=\frac{\Psi^{\prime}(\beta)}{\Psi(\beta)}$. Calculations show that this function is decreasing on the interval $\left(0 ; \beta_{0}\right]$ and increases on $\left[\beta_{0} ; \sqrt{2}\right)$, $0.85<\beta_{0}<1$. Further, as in the proof of Theorem 6 in [5], we verify that the unique solution of the extremal problem is the point $\left(\frac{\sqrt{2}}{n}, \ldots, \frac{\sqrt{2}}{n}\right)$. Estimates (4), (5), (8), (9) yield the inequality (3) of Theorem 2. The case of equality is verified directly. Theorem 2 is proved.

Proof of Theorem 3. The proof is based on application of separating transformation, developed in details in [6]. According to the conditions of Theorem 3, $a_{0}=0,1-\varepsilon<\left|a_{k}\right|<1+\varepsilon, k=\overline{1,2}$. Assume

$$
0=\arg a_{1}<\arg a_{2}<2 \pi .
$$

Let $\alpha_{1}:=\frac{1}{\pi} \cdot\left(\arg a_{2}-\arg a_{1}\right), \alpha_{2}:=\frac{1}{\pi} \cdot\left(2 \pi-\arg a_{2}\right), P_{k}:=\left\{w: \arg a_{k}<\right.$ $\left.\arg w<\arg a_{k+1}\right\}, k=\overline{1,2}, \arg a_{3}=2 \pi, P_{0}:=P_{2}, P_{3}:=P_{1}$.

The family of two symmetrical domains $\left\{D_{k}^{(1)} ; D_{k-1}^{(2)}\right\}$ with respect to the imaginary axis is called a result of separating transformation of the domain $B_{k}$. 
Further, as in Theorem 5.2.1 in [3], using the separating transformation we obtain

$$
J_{2}(\gamma) \leq \mathcal{L}^{(\gamma)}\left(A_{2}\right)\left[\prod_{k=1}^{2} \alpha_{k}^{2} r^{\gamma \cdot \alpha_{k}^{2}}\left(D_{0}, 0\right) \cdot r\left(D_{1}, 1\right) \cdot r\left(D_{2},-1\right)\right]^{\frac{1}{2}} .
$$

We will prove that for $\alpha_{0} \geq \frac{2}{\sqrt{\gamma}}, \alpha_{0}=\max \left\{\alpha_{1}, \alpha_{2}\right\}$ extremal configurations different than those referred in Theorem 3 do not exist. For this we find a value of the functional (see Theorem 5.2.3 in [3])

$$
J_{2}^{0}(\gamma)=r^{\gamma}\left(D_{0}, 0\right) \cdot \prod_{k=1}^{2} r\left(D_{k}, d_{k}\right)=4 \cdot \frac{(\gamma)^{\frac{\gamma}{2}}}{\left(1-\frac{\gamma}{4}\right)^{2+\frac{\gamma}{2}}} \cdot\left(\frac{1-\frac{\sqrt{\gamma}}{2}}{1+\frac{\sqrt{\gamma}}{2}}\right)^{2 \sqrt{\gamma}}
$$

if $\gamma=1.1$. We have that $J_{2}^{0}(1.1) \approx 0.8315$.

Denote $r\left(B_{0}, 0\right)=r_{0}, r\left(B_{1}, a_{1}\right)=r_{1}, r\left(B_{2}, a_{2}\right)=r_{2}$. The Lavrent'ev's theorem [17] gives $r_{0} r_{1}<\left|a_{1}\right|^{2}, r_{0} r_{2}<\left|a_{2}\right|^{2}, r_{0}^{2} r_{1} r_{2}<\left|a_{1}\right|^{2}\left|a_{2}\right|^{2} \Longrightarrow r_{1} r_{2}<$ $\frac{\left|a_{1}\right|^{2}\left|a_{1}\right|^{2}}{r_{0}^{2}}$.

Then $r^{\gamma}\left(B_{0}, a_{0}\right) \cdot \prod_{k=1}^{2} r\left(B_{k}, a_{k}\right)=r_{0}^{\gamma} \cdot \prod_{k=1}^{2} r\left(B_{k}, a_{k}\right) \leq r_{0}^{\gamma} \cdot \frac{\left|a_{1}\right|^{2} \cdot\left|a_{2}\right|^{2}}{r_{0}^{2}} \leq$ $J_{2}^{0}(\gamma) \Rightarrow r_{0} \geq\left(\frac{\left|a_{1}\right|^{2} \cdot\left|a_{2}\right|^{2}}{J_{2}^{0}(\gamma)}\right)^{\frac{1}{2-\gamma}}$. If $r_{0} \geq\left(\frac{\left|a_{1}\right|^{2} \cdot\left|a_{2}\right|^{2}}{J_{2}^{0}(\gamma)}\right)^{\frac{1}{2-\gamma}}$, then the extremal configurations do not exist. Consider the case $r_{0} \leq\left(\frac{\left|a_{1}\right|^{2} \cdot\left|a_{2}\right|^{2}}{J_{2}^{0}(\gamma)}\right)^{\frac{1}{2-\gamma}}$.

$$
\begin{aligned}
& J_{2}(\gamma) \leq r_{0}^{\gamma}\left|a_{1}-a_{2}\right|^{2}=r_{0}^{\gamma}\left(\left(\left|a_{1}\right|-\left|a_{2}\right|\right)^{2}+4\left|a_{1}\right| \cdot\left|a_{2}\right| \sin ^{2}\left(2-\alpha_{0}\right) \frac{\pi}{2}\right) \\
& \leq\left(\frac{\left|a_{1}\right| \cdot\left|a_{2}\right|}{J_{2}^{0}(\gamma)}\right)^{\frac{\gamma}{2-\gamma}} \cdot\left(\left(\left|a_{1}\right|-\left|a_{2}\right|\right)^{2}+4\left|a_{1}\right| \cdot\left|a_{2}\right| \sin ^{2}\left(2-\alpha_{0}\right) \frac{\pi}{2}\right) \leq J_{2}^{0}(1.1) .
\end{aligned}
$$

Substituting $\varepsilon=0.25, \gamma=1.1, n=2,\left|a_{1}\right|=1-\varepsilon,\left|a_{2}\right|=1+\varepsilon$, $J_{2}^{0}(1.1)=0.8315$ in the last inequality, we obtain

$$
(1+\varepsilon)^{\frac{1.1}{2-1,1}}\left(4 \varepsilon^{2}+4(1+\varepsilon) \sin ^{2}\left(2-\frac{2}{\sqrt{1.1}}\right) \frac{\pi}{2}\right) \leq J_{2}^{0}(1.1)^{1+\frac{1.1}{2-1.1}}
$$

Performing calculations of right and left sides of the inequality (11), we have $0.6085<0.6663$. From this it follows that if $\varepsilon=0.25$, then inequality (11) is true. Hence $J=\frac{J_{2}(1.1)}{J_{2}^{0}(1.1)} \leq \frac{0.7316}{0.8315}=0.8798<1$, i.e. if $\alpha_{0}>\frac{2}{\sqrt{\gamma}}$, $J_{2}(\gamma)<J_{2}^{0}(\gamma)$ then the maximum value of the functional $J_{2}(\gamma)$ for such domains is not attained. Then $\alpha_{0} \leq \frac{2}{\sqrt{\gamma}}$ and we can apply inequality (10).

Using a result obtained in the proof of Theorem 4 in [5], we can write the following inequality

$$
J_{2}(\gamma) \leq \frac{1}{\sqrt{\gamma}} \cdot\left[\prod_{k=1}^{2} 2^{\sigma_{k}^{2}+6} \cdot \sigma_{k}^{\sigma_{k}^{2}+2} \cdot\left(2-\sigma_{k}\right)^{-\frac{1}{2}\left(2-\sigma_{k}\right)^{2}}\left(2+\sigma_{k}\right)^{-\frac{1}{2}\left(2+\sigma_{k}\right)^{2}}\right]^{\frac{1}{2}},
$$


where $\sigma_{k}=\sqrt{\gamma} \cdot \alpha_{k}$. Consider the function

$$
\Psi(\sigma)=2^{\sigma^{2}+6} \cdot \sigma^{\sigma^{2}+2} \cdot(2-\sigma)^{-\frac{1}{2}(2-\sigma)^{2}}(2+\sigma)^{-\frac{1}{2}(2+\sigma)^{2}},
$$

$\sigma \in[0,2]$ and we will conduct detailed investigation of its graph on this interval (see Fig.1).

$\Psi(\sigma)$ is logarithmically convex on interval $\left[0 ; x_{0}\right], x_{0} \approx 1.32$. On $\left[0 ; x_{1}\right]$, $x_{1} \approx 1.05$ the function increases from $\Psi(0)=0$ to $\Psi\left(x_{1}\right) \approx 0.9115$, and it decreases on interval $\left[x_{1} ; x_{2}\right], x_{2} \approx 1.6049$ to $\Psi\left(x_{2}\right) \approx 0.86$, and on $\left[x_{2} ; 2\right]$ it increases to $\Psi(2)=1 . x_{3} \approx 1.9, \Psi\left(x_{3}\right)=\Psi\left(x_{1}\right) \approx 0.9115$.

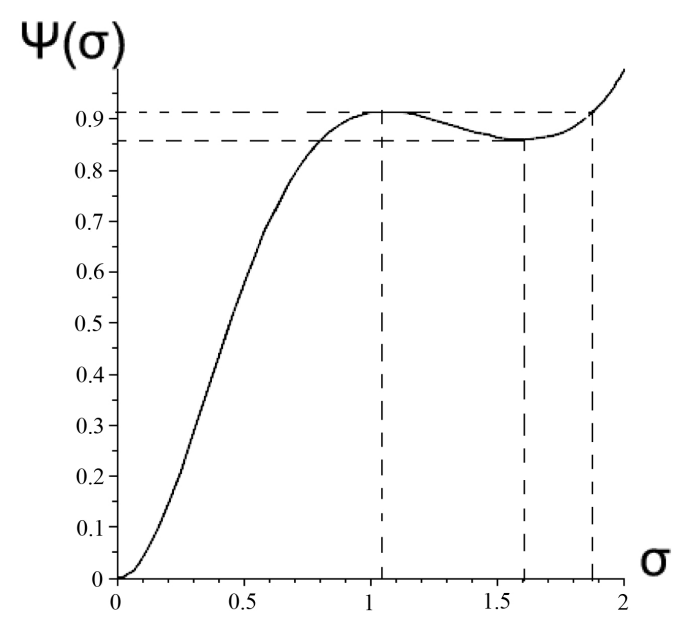

FiguRE 1.

Using equality $\sigma_{1}+\sigma_{2}=2 \sqrt{\gamma}$, we will prove that $\Psi\left(\sigma_{1}\right) \cdot \Psi\left(\sigma_{2}\right) \leq$ $\left(\Psi\left(x_{1}\right)\right)^{2} \approx 0.8308$. For $\sigma \in\left[0 ; x_{0}\right]$ we make appropriate conclusion from the logarithmic convexity of the function $\Psi(\sigma)$. For $\sigma_{2} \in\left[x_{0} ; x_{3}\right]$ from properties of the graph of the function $\Psi(\sigma)$, we have $\Psi\left(\sigma_{2}\right) \leq \Psi\left(x_{1}\right)$ and $\Psi\left(\sigma_{1}\right) \leq \Psi\left(x_{1}\right)$ and thus $\Psi\left(\sigma_{1}\right) \cdot \Psi\left(\sigma_{2}\right) \leq\left(\Psi\left(x_{1}\right)\right)^{2}$.

If $\sigma_{2} \in\left[x_{3} ; 2\right]$ then $\Psi\left(\sigma_{2}\right)<\Psi(2)=1, \Psi\left(\sigma_{1}\right)<\Psi(0,2) \ll 0.4$, and hence $\Psi\left(\sigma_{1}\right) \cdot \Psi\left(\sigma_{2}\right)<0,4<\left(\Psi\left(x_{1}\right)\right)^{2}$. So, $J_{2}(\gamma) \leq J_{2}^{0}(\gamma)$. Inequality (11) is true and Theorem 3 is proved.

Acknowledgement. The author is grateful to Prof. Bakhtin for suggesting problems and useful discussions.

\section{REFERENCES}

[1] Bieberbach, L., Über die Koeffizienten derjenigen Potenzreihen, welche eine schlichte Abbildung des Einheitskreises vermitteln, Sitzungsber. Preuss. Akad. Wiss. PhysMath. Kl. 138 (1916), 940-955. 
[2] Bakhtin, A. K., Bakhtina, G. P., Separating transformation and problem on nonoverlapping domains, Proceedings of Institute of Mathematics of NAS of Ukraine 3 (4) (2006), 273-281.

[3] Bakhtin, A. K., Bakhtina, G. P., Zelinskii, Yu. B., Topological-algebraic structures and geometric methods in complex analysis, Proceedings of the Institute of Mathematics of NAS of Ukraine $\mathbf{7 3}$ (2008), 308 pp. (Russian).

[4] Dubinin, V. N., The symmetrization method in problems on non-overlapping domains, Mat. Sb. (N.S.) 128 (1) (1985), 110-123 (Russian).

[5] Dubinin, V. N., A separating transformation of domains and problems on extremal decomposition, Zap. Nauchn. Sem. Leningrad. Otdel. Mat. Inst. Steklov. (LOMI) 168 (1988), 48-66 (Russian); translation in J. Soviet Math. 53, no. 3 (1991), 252-263.

[6] Dubinin, V. N., Symmetrization method in geometric function theory of complex variables, Uspekhi Mat. Nauk 49, no. 1 (1994), 3-76 (Russian); translation in Russian Math. Surveys 49, no. 1 (1994), 1-79.

[7] Dubinin, V. N., Asymptotics of the modulus of a degenerate condenser, and some of its applications, Zap. Nauchn. Sem. S.-Peterburg. Otdel. Mat. Inst. Steklov. (POMI) 237 (1997), 56-73 (Russian); translation in J. Math. Sci. (New York) 95, no. 3 (1999), 2209-2220.

[8] Dubinin, V. N., Capacities of condensers and symmetrization in geometric function theory of complex variables, Dal'nayka, Vladivostok, 2009 (Russian).

[9] Duren, P. L., Univalent Functions, Springer-Verlag, New York, 1983.

[10] Goluzin, G. M., Geometric Theory of Functions of a Complex Variable, Translations of Mathematical Monographs, no. 26, Amer. Math. Soc., Providence, R.I. (1969).

[11] Grötzsch, H., Über einige Extremalprobleme der konformen Abbildung. I, II, Ber. Verh. Sächs. Akad. Wiss. Leipzig, Math.-Phys. 80 (6) (1928), 367-376, 497-502.

[12] Grunsky, H., Koeffizientenbedingungen für schlicht abbildende meromorphe Funltionen, Math. Z. 45, no. 1 (1939), 29-61.

[13] Hayman, W. K., Multivalent Functions, Cambridge University Press, Cambridge, 1958.

[14] Jenkins, J. A., Some uniqueness results in the theory of symmetrization, Ann. Math. 61, no. 1 (1955), 106-115.

[15] Kolbina, L. I., Conformal mapping of the unit circle onto non-overlapping domains, Vestnik Leningrad. Univ. 10, no. 5 (1955), 37-43 (Russian).

[16] Kovalev, L. V., On the problem of extremal decomposition with free poles on the circle, Dal'nevost. Mat. Sb. 2 (1996), 96-98 (Russian).

[17] Lavrent'ev, M. A., On the theory of conformal mappings, Tr. Fiz.-Mat. Inst. Akad. Nauk SSSR, Otdel. Mat. 5 (1934), 195-245 (Russian).

[18] Nehari, Z., Some inequalities in the theory of functions Trans. Amer. Math. Soc. 75, no. 2 (1953), 256-286.

[19] Riemann, B., Theorie der Abelschen Functionen J. Reine Angew. Math. 54 (1867), 101-155.

[20] Teichmüller, O., Collected Papers, Springer, Berlin, 1982.

[21] Vasil'ev, A., Moduli of Families of Curves for Conformal and Quasiconformal Mappings, Springer-Verlag, Berlin, 2002. 
Iryna V. Denega

Department of Complex Analysis and Potential Theory

Institute of Mathematics of National Academy of Sciences of Ukraine

Tereshchenkivska St. 3

01601 Kyiv

Ukraine

e-mail: iradenega@yandex.ru

Received September 20, 2011 